\title{
Eficacia del ácido tranexámico para disminuir el sangrado perioperatorio en artroplastía total reversa primaria
}

\author{
Efficacy of Tranexamic Acid to decrease perioperative \\ bleeding in primary total reverse arthroplasty
}

\author{
Palmieri-Bouchan RB,* Aguirre-Rodríguez VH, ${ }^{\star}$ Salinas-Vela LE, ${ }^{*}$ Encalada-Díaz MI,* Valero-González FS \\ Clínica de Reconstrucción Articular de Hombro y Codo. Hospital Ángeles Pedregal.
}

RESUMEN. Introducción: La artroplastía reversa de hombro mejora la función y el dolor en pacientes con artrosis glenohumeral y deficiencia del manguito de los rotadores. Sin embargo, se asocia con pérdida sanguínea significativa y formación de hematoma en el postoperatorio (PO). Objetivo: Determinar la eficacia del ácido tranexámico (ATXM) para disminuir el sangrado perioperatorio en pacientes sometidos a artroplastía reversa primaria de hombro. Material y métodos: Cohorte de 31 pacientes sometidos a artroplastía primaria reversa, dos grupos: grupo A (17 pacientes) se les aplicó $1 \mathrm{~g}$ intravenoso de ácido tranexámico preoperatorio y el grupo B (14 pacientes) a quienes no se les administró. Se registraron la hemoglobina y el hematocrito preoperatorio y a las 24 horas de la cirugía y el sangrado cuantificado en el drenaje a las 24 horas, así como las necesidades de transfusión sanguínea. Se compararon estadísticamente los cambios presentados. Resultados: Los datos demográficos no demostraron diferencias significativas entre ambos grupos. Hay tendencia a menor sangrado en el drenaje quirúrgico en los pacientes con ATXM (115.6 vs $162.65 \mathrm{ml}, \mathrm{p}=0.0768)$, en la disminución de hemoglobina (2.16 vs $2.79 \mathrm{~g} / \mathrm{dl}, \mathrm{p}=0.1257)$ y del hematocrito $(6.67 \%$ vs $7.95 \% p=0.3431)$. Existió una significativa reducción en el uso de hemoderivados en los pacientes con ATXM ( $p$ $=0.0441$ ). Conclusiones: Existe una tendencia a presentar
ABSTRACT. Introduction: Reverse shoulder arthroplasty improves function and pain in patients with glenohumeral osteoarthritis and rotator cuff deficiency. However, it is associated with significant blood loss and postoperative hematoma (PO) formation. Objective: To determine the efficacy of tranexamic acid (ATXM) to decrease perioperative bleeding in patients undergoing primary reverse shoulder arthroplasty. Material and methods: Cohort of 31 patients undergoing reverse primary arthroplasty, in two groups: group A (17 patients) were given $1 \mathrm{~g}$ IV of preoperative tranexamic acid and group B (14 patients) to whom it was not administered. Preoperative hemoglobin and hematocrit were recorded and then after 24 hours of surgery, and quantified bleeding in drainage at 24 hours, as well as blood transfusion needs. The changes presented were statistically compared. Results: Demographic data did not show significant differences between the two groups. There is a tendency to reduce bleeding in surgical drainage in patients with ATXM (115.6 $\mathrm{ml}$ vs. $162.65 \mathrm{ml} \mathrm{p}=0.0768)$, in the decrease of hemoglobin $(2.16 \mathrm{~g} / \mathrm{dl}$ vs. $2.79 \mathrm{~g} / \mathrm{dl} \mathrm{p}=0.1257)$ and hematocrit $(6.67 \%$ vs. $7.95 \% p=0.3431)$. There was a significant reduction in the use of blood products in patients with ATXM ( $\mathrm{p}=$ 0.0441 ). Conclusion: There is a tendency to have less PO bleeding in patients undergoing primary reverse arthroplasty

\section{Nivel de evidencia: IV. Estudio retrospectivo comparativo}

\footnotetext{
* Alumno del Curso.

* Profesor Asociado del Curso.

$\S$ Profesor Titular del Curso.
}

Alta Especialidad en Cirugía de Reconstrucción Articular de Hombro y Codo. Facultad Mexicana de Medicina de la Universidad La Salle. Hospital Ángeles Pedregal, GASS. México.

Correspondencia:

Dr. Fernando Sergio Valero-González

Camino a Santa Teresa 1055-970, Col. Héroes de Padierna, CP 10700, Alcaldía Magdalena Contreras, CDMX.

E-mail: shoulder.elbow@gmail.com

Citar como: Palmieri-Bouchan RB, Aguirre-Rodríguez VH, Salinas-Vela LE, Encalada-Díaz MI, Valero-González FS. Eficacia del ácido tranexámico para disminuir el sangrado perioperatorio en artroplastía total reversa primaria. Acta Ortop Mex. 2020; 34(5): 288-292. https://dx.doi.org/10.35366/97990

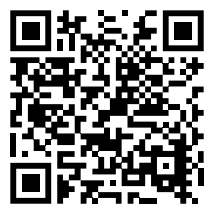


menor sangrado PO en pacientes sometidos a artroplastía reversa primaria con el uso del ATXM y un menor requerimiento de transfusión sanguínea PO.

Palabras clave: Ácido tranexámico, artroplastía reversa, artroplastía de hombro, complicaciones, sangrado.

\section{Introducción}

La artroplastía reversa de hombro es la mejor opción para recuperar la función y disminuir el dolor en pacientes con artrosis glenohumeral severa y deficiencia del manguito de los rotadores no reparable. Sin embargo, al igual que en otros casos de reemplazo articular, se asocia con una pérdida sanguínea significativa y formación de hematomas en el PO. La artroplastía reversa de hombro es un factor de riesgo independiente para transfusión sanguínea, ${ }^{1}$ esta última se asocia con efectos adversos como reacciones inmunológicas, hemolisis intravascular, falla renal, lesión pulmonar y costos hospitalarios mayores. ${ }^{2}$

El ácido tranexámico es un agente antifibrinolítico derivado de la lisina que inhibe de forma reversible los sitios de unión de la lisina en las moléculas del plasminógeno y, con esto, su activación en plasmina impidiendo la degradación de fibrina y disminuyendo el sangrado perioperatorio. ${ }^{3} \mathrm{Su}$ uso ha sido probado ampliamente en artroplastía de rodilla y cadera, sin embargo, pocos estudios se han realizado para validar su uso en reemplazo articular de hombro y a nuestro saber ninguno en población latinoamericana.

El objetivo primario de este estudio es determinar la eficacia del ácido tranexámico para disminuir el sangrado perioperatorio y el requerimiento de transfusión sanguínea en pacientes sometidos a artroplastía total de hombro con implante reverso, la hipótesis propuesta fue que los

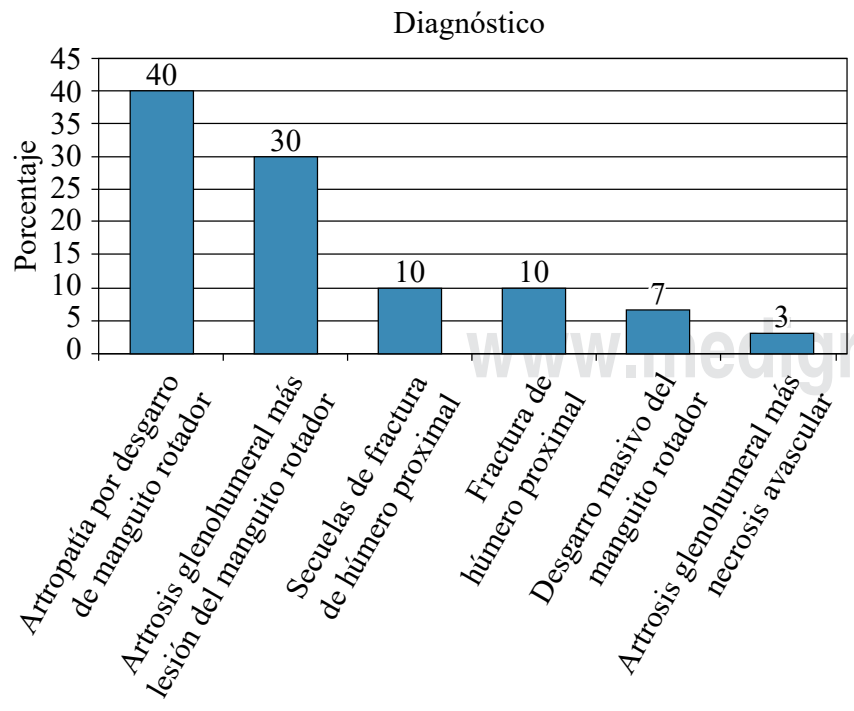

Figura 1: Distribución por diagnósticos $(\mathrm{N}=32)$. with the use of ATXM and a lower PO blood transfusion requirement.

Keywords: Tranexamic acid, reverse replacement, shoulder, complications, bleeding.

pacientes que recibieron ATXM preoperatorio experimentaron un menor sangrado, una caída menor en los valores de hemoglobina y hematocrito postoperatorios y además presentaron menores requerimientos de transfusión postoperatoria $(\mathrm{PO})$.

\section{Material y métodos}

Se realizó un análisis retrospectivo, comparativo, observacional, descriptivo y longitudinal. Se incluyeron pacientes de ambos géneros, entre 55 y 90 años, intervenidos quirúrgicamente mediante artroplastía primaria de hombro con implante reverso, desde el 01 de Abril del 2014 al 01 de Agosto del 2019 en esta institución. De los 74 registros médicos revisados, cumplieron los criterios de inclusión 31 pacientes, de los cuales uno fue sometido a artroplastía de ambos hombros obteniéndose un total de 32 procedimientos a partir de los cuales se establecieron dos cohortes, el grupo A con pacientes a los que se les aplicó $1 \mathrm{~g}$ intravenoso preoperatorio de ATXM y el grupo B con pacientes a los que no se les aplicó. En ambos grupos se registraron la hemoglobina y el hematocrito preoperatorio y a las 24 horas de la cirugía y el sangrado cuantificado en el drenaje a las 24 horas del procedimiento, de igual forma, se registraron los pacientes que tuvieron necesidad de ser transfundidos por indicación de presentar hemoglobina menor a $9 \mathrm{~g} / \mathrm{dl}$ y repercusión hemodinámica durante su estancia hospitalaria.

\begin{tabular}{|c|c|c|c|}
\hline & $\begin{array}{l}\text { Grupo A } \\
(\mathrm{n}=18)\end{array}$ & $\begin{array}{l}\text { Grupo B } \\
(\mathrm{n}=14)\end{array}$ & \\
\hline & $\begin{array}{l}\text { Con ácido } \\
\text { tranexámico }\end{array}$ & $\begin{array}{l}\text { Sin ácido } \\
\text { tranexámico }\end{array}$ & $\mathrm{p}$ \\
\hline Género & F $13 / \mathrm{M} 4$ & F 9/M 5 & 0.999 \\
\hline DM2 & 1 & 1 & 1.000 \\
\hline HAS & 7 & 8 & 0.999 \\
\hline Cardiopatía & 0 & 2 & 0.855 \\
\hline Edad & 70.94 & 71.50 & 0.845 \\
\hline $\begin{array}{l}\mathrm{Hb} \\
\text { preoperatorio }\end{array}$ & 14.42 & 14.93 & 0.351 \\
\hline $\begin{array}{l}\text { Hcto } \\
\text { preoperatorio }\end{array}$ & 43.62 & 44.79 & 0.223 \\
\hline
\end{tabular}



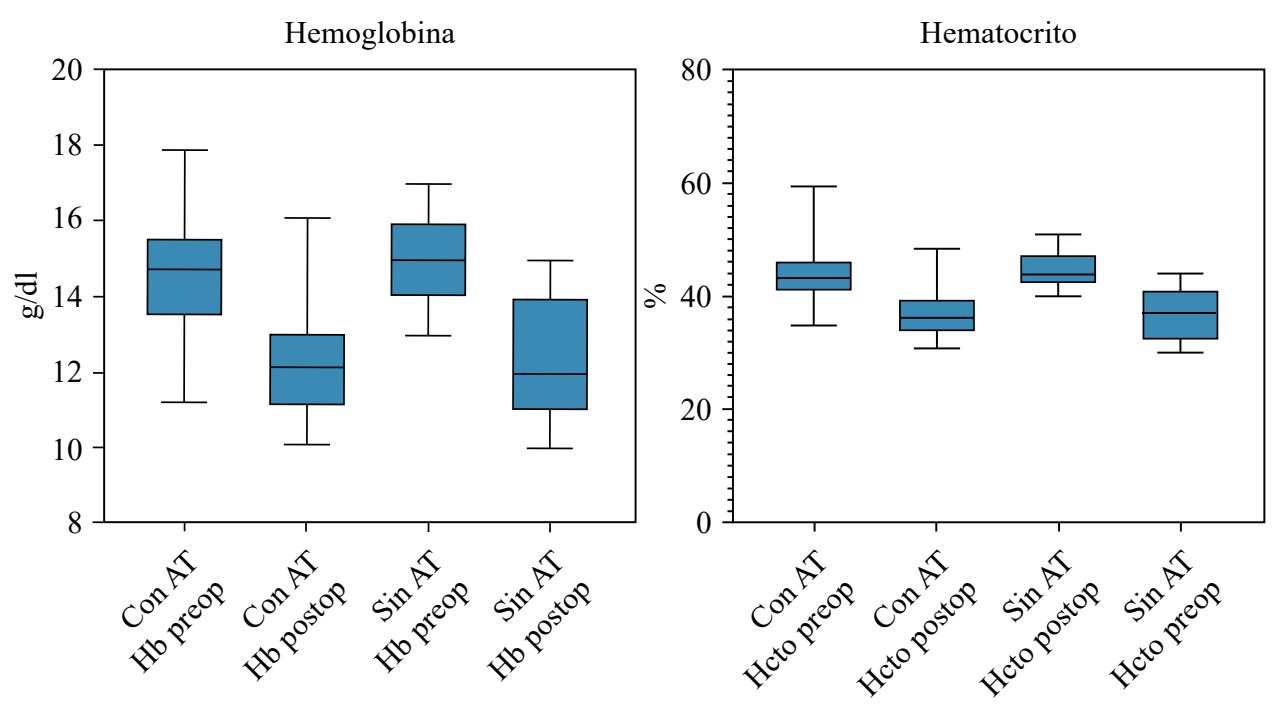

Figura 2:

Valores pre/postoperatorios de hemoglobina y hematocrito en ambos grupos.

Con $\mathrm{AT}=$ Con ácido tranexámico; Sin $\mathrm{AT}=$ sin ácido tranexámico; $\mathrm{Hb}$ preop $=$ hemoglobina preoperatoria; $\mathrm{Hb}$ postop $=$ hemoglobina postoperatoria; Hcto preop $=$ hematocrito preoperatorio; Hcto postop $=$ hematocrito postoperatorio.

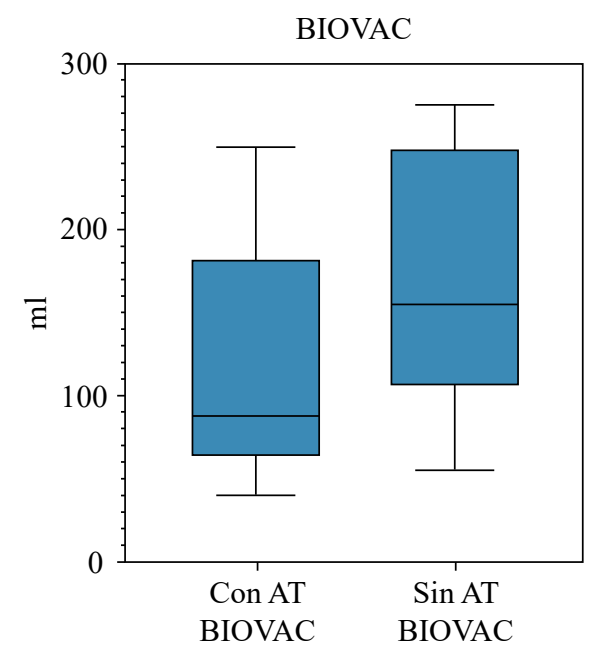

Figura 3: Sangrado registrado a las 24 horas postoperatorias. Grupo A: media $115.6 \mathrm{ml}$ (DE: $67.28 \mathrm{ml}$ ). Grupo B: media $162.65 \mathrm{ml}$ (DE: 72.45 $\mathrm{ml})(\mathrm{p}=0.0768)$.

El análisis estadístico se llevó a cabo mediante la prueba $\mathrm{t}$ de Student para los cambios presentados en la hemoglobina y el hematocrito y el sangrado a las 24 horas y a través de la prueba $\chi^{2}$ la necesidad de transfusión sanguínea en el postoperatorio. Se estableció un nivel de confianza de $95 \%$ $\mathrm{y}$ un valor de $\mathrm{p}<0.05$ fue considerado significativo.

Todas las cirugías fueron realizadas por un solo cirujano (FSVG). Al ingreso al quirófano los pacientes del grupo A recibieron $1 \mathrm{~g}$ de ATXM intravenoso. Todos los pacientes recibieron bloqueo interescalénico más anestesia general y fueron intervenidos a través de un abordaje deltopectoral con hemostasia meticulosa. los implantes utilizados fueron: Delta Xtend (DePuy Synthes, Warsaw, IN, USA) en 29 pacientes y Comprehensive Reverse Shoulder system (Zimmer-Biomet, Warsaw, IN, USA) en dos pacientes, al término del procedimiento se colocó un drenaje Biovac (Biometrix, $\mathrm{OH}, \mathrm{USA}$ ), el cual fue cuantificado y registrado de forma continua durante su hospitalización. Al día siguiente al

procedimiento se obtuvieron valores postoperatorios de hemoglobina y hematocrito determinándose con estas cifras y las condiciones clínicas (repercusión hemodinámica) la necesidad de transfusión de hemoderivados.

\section{Resultados}

La muestra incluyó 32 procedimientos (en 31 pacientes), de los cuales 18 (56.25\%) recibieron ATXM preoperatorio y $14(47.75 \%)$ no. La indicación de la artroplastía reversa se muestra en la Figura 1, siendo la artropatía por desgarro de manguito rotador más frecuente $(40 \%)$. La media de edad fue de 71.20 años (rango 58-88 años). Fueron 22 pacientes mujeres (70.96\%) y nueve hombres (29.03\%). El 57\% de los pacientes fueron sometidos a cirugía del lado derecho.

Al realizar el análisis multivariado de los datos demográficos de ambos grupos no se demostraron diferencias estadísticamente significativas (Tabla 1).

No existió diferencia estadísticamente significativa entre ambos grupos en los valores de hemoglobina y hematocrito pre y postoperatorios. Estos se muestran en la Figura 2.

Se cuantificó el drenaje quirúrgico a las 24 horas $\mathrm{PO}$ en ambos grupos observándose una tendencia a menor sangrado en pacientes tratados con ATXM $(\mathrm{p}=0.0768)$ (Figura 3$)$.

En la Tabla 2 se muestra la variación pre/postoperatoria de la hemoglobina y hematocrito. Los requerimientos de transfusión sanguínea fueron registrados y se muestran en la Figura 4, presentando una diferencia significativa entre ambos grupos $(\mathrm{p}=0.0441)$.

\section{Discusión}

La artroplastía total de hombro es considerada un procedimiento asociado con sangrado importante tanto en el transoperatorio como en el postoperatorio, reportándose $2.6 \%$ de hematomas postquirúrgicos; ${ }^{4}$ en la búsqueda de disminuir el sangrado perioperatorio y con ello las complicaciones por requerimientos de transfusiones de hemoderivados, se han 
tomado múltiples medidas como hipotensión controlada, hemodilución, recuperadores celulares intraoperatorios y, más recientemente, la introducción de quimioprofilácticos antifibrinolíticos como el ácido tranexámico. ${ }^{5,6}$

El ATXM es un medicamento generalmente bien tolerado, cuyas propiedades antifibrinolíticas disminuyen los hematomas quirúrgicos, producen un menor sangrado quirúrgico y menores tasas de transfusión sanguínea en cirugía de columna y artroplastías de rodilla y cadera. ${ }^{7,8}$ Con el aumento en la realización de artroplastías de hombro y especialmente del uso de prótesis reversa en los últimos años, la necesidad de buscar opciones para disminuir las complicaciones transoperatorias ha llevado a la realización de diversos estudios para comprobar la efectividad del ATXM en estos procedimientos. ${ }^{9,10}$

Existen pocos reportes acerca del valor del ATXM en la cirugía de artroplastía de hombro comparado con lo encontrado en los reemplazos de cadera y rodilla. En 2015, Gillespie y colaboradores en un estudio prospectivo aleatorizado demostraron que la aplicación de ATXM tópico disminuyó el sangrado postoperatorio ( $170 \mathrm{vs} 108 \mathrm{ml})$ y la caída en los valores de hemoglobina ( $2.6 \mathrm{vs} 1.7 \mathrm{~g} / \mathrm{dl}$ ) en pacientes sometidos a artroplastía anatómica y reversa de hombro. ${ }^{11}$ Friedman y colaboradores realizaron un estudio retrospectivo utilizando ATXM intravenoso en artroplastía total anatómica o reversa, encontrando que los pacientes a los que se les aplicó tuvieron menos cambios en los valores de hemoglobina y hematocrito, $21 \%$ menos tiempo en recuperación postoperatoria, $16 \%$ menos estancia hospitalaria. ${ }^{12}$

En 2017, Vara y su equipo llevaron a cabo un estudio prospectivo, controlado, aleatorizado, doble ciego en 102 pacientes, en el que comprobaron que la aplicación de ATXM intravenoso disminuye de forma efectiva la pérdida de hemoglobina y el sangrado perioperatorio, así como la posibilidad de requerir transfusión sanguínea intrahospitalaria. ${ }^{13}$ En el mismo año, Pauzenberger y cols. analizaron de forma prospectiva la aplicación de $1 \mathrm{~g}$ de ATXM intravenoso en 27 pacientes sometidos a artroplastía total primaria reversa contra 27 pacientes a quienes no se les aplicó y encontraron que en el primer grupo se presentó un menor san-

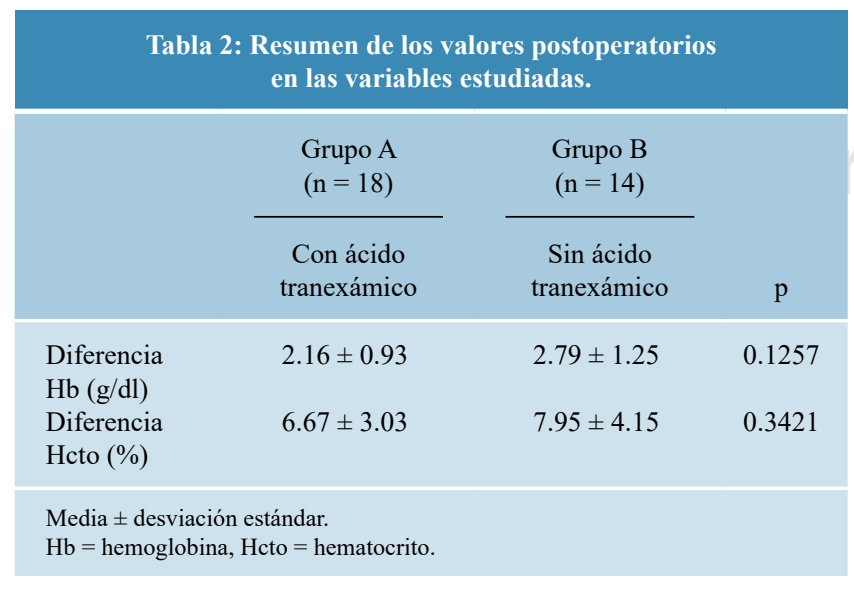

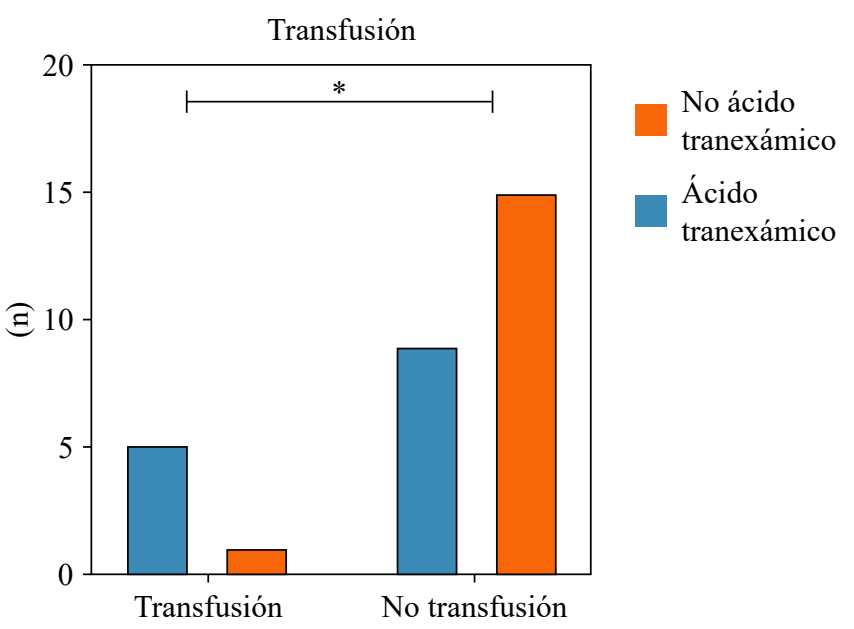

Figura 4: Necesidades de transfusión en ambos grupos. Existió una diferencia a menos transfusiones sanguíneas en pacientes con ATXM.

grado cuantificado en el drenaje quirúrgico (170 vs $50 \mathrm{ml}$ ), menor formación de hematoma postoperatorio $(59.3 \%$ vs $25.9 \%$ ) y un mejor control analgésico (EVA: 2.0 vs 1.3 ). ${ }^{14}$

Anthony y colaboradores, en un estudio retrospectivo con 1922 casos de artroplastía de hombro, encontraron que la complicación más frecuente fue el sangrado postoperatorio que requirió transfusión de hemoderivados alcanzando hasta $4.26 \%$ de todas las complicaciones. ${ }^{1}$ No obstante, se ha llegado a documentar en la literatura necesidades de transfusión hasta en $43 \% .^{15}$

En 2018, Kuo y asociados realizaron una revisión sistemática incluyendo estudios nivel I y III de evidencia, concluyendo que el riesgo de requerir una transfusión sanguínea en artroplastía de hombro es menor en pacientes en los que se utilizó ATXM (7/319 vs 20/313 pacientes con p < $0.00001) .{ }^{16}$ Finalmente, en 2019 se llevó a cabo el primer estudio a larga escala utilizando la base de datos «Premier Healthcare» donde se incluyeron 82,512 registros médicos para valorar la efectividad del ácido tranexámico en disminuir las complicaciones perioperatorias, encontrando que su uso en dosis de $1 \mathrm{~g}$ preoperatorio reduce en $36 \%$ el riesgo de requerir transfusión sanguínea $(\mathrm{p}<0.05){ }^{1}$

Los hallazgos del presente estudio concuerdan con lo encontrado en la literatura internacional ya que, a pesar de no haber alcanzado un valor de $\mathrm{p}<0.05$ en todas las variables estudiadas, los valores del sangrado postoperatorio medido en el drenaje quirúrgico, los cambios entre la hemoglobina y el hematocrito pre y postoperatorios mostraron una tendencia a favor del uso de ATXM intravenoso en pacientes sometidos a artroplastía total primaria de hombro con implante reverso. Así mismo, el hecho de que el número de pacientes transfundidos fue mayor en el grupo B que en el grupo A, implica una reducción en las complicaciones - en este caso el sangrado-y disminución de los gastos y riesgos derivados de la transfusión, como lo muestran los trabajos de Anthony CA, ${ }^{1}$ Spiess $\mathrm{BD}^{2}$ y Boileau P. ${ }^{4}$

De manera similar a este estudio, Kim y cols. buscaron corroborar la efectividad del ATXM intravenoso en pobla- 
ción no caucásica para disminuir el sangrado y la caída de los valores sanguíneos en 24 pacientes sometidos a artroplastía reversa, encontrando una diferencia significativa en los valores de hemoglobina, hematocrito y sangrado postoperatorio medidos a las 48 horas después de la cirugía, a favor de los pacientes con ATXM. ${ }^{18}$ A pesar de contar con un tamaño de muestra similar, es probable que la diferencia con este estudio se relacione con la determinación de estas variables hasta las 48 horas.

Abildgaard y cols., en un análisis de regresión multivariado en 168 pacientes, encontraron que factores como la edad y el sexo del paciente se asocian con un aumento en el sangrado postoperatorio. ${ }^{19}$ Contrario a sus hallazgos, no se encontró una relación entre los factores demográficos del paciente y una mayor posibilidad de sangrado en artroplastía reversa pudiendo relacionarse a un menor tamaño de muestra en el presente trabajo.

Existen varias limitaciones en este estudio, entre ellas su diseño retrospectivo, sin embargo, el que los protocolos utilizados antes y después del procedimiento hayan sido idénticos en todos los pacientes, así como el haber sido realizado por un único cirujano disminuyen los sesgos en la investigación. Otra limitación es el tamaño de muestra, que es menor si se compara con los números mostrados en algunos estudios internacionales, no obstante, es importante reconocer que la artroplastía reversa de hombro en México y Latinoamérica es aún una cirugía con volúmenes bajos.

Una de las fortalezas de este trabajo es que no existe en este momento otro reporte similar en población latinoamericana. Esta investigación puede dar inicio al desarrollo de estudios clínicos aleatorizados para validar estos hallazgos iniciales obtenidos por el equipo médico de los autores, respecto al uso de ATXM en artroplastía de hombro para disminuir el sangrado postoperatorio y sus complicaciones.

Esta sencilla medida puede mejorar el resultado al disminuir una de las complicaciones más frecuentes de este procedimiento y los riesgos inherentes al uso de hemoderivados, por lo que debe considerarse como parte de los protocolos quirúrgicos en centros de cirugía de hombro, especialmente en pacientes con alto riesgo de transfusión sanguínea.

\section{Conclusiones}

El ácido tranexámico intravenoso aplicado de forma preoperatoria puede disminuir el sangrado en pacientes sometidos a artroplastía primaria con implante reverso.

Referencias

1. Anthony CA, Westermann RW, Gao Y, Pugely AJ, Wolf BR, Hettrich $\mathrm{CM}$. What are risk factors for 30-day morbidity and transfusion in total shoulder arthroplasty? A review of 1922 cases. Clin Orthop Relat Res. 2015; 473: 2099-105.

2. Spiess BD. Risks of transfusion: outcome focus. Transfusion. 2004; 44(12 Suppl): 4S-14S.

3. Dunn CJ, Goa KL. Tranexamic acid: a review of its use in surgery and other indications. Drugs. 1999; 57: 1005-32.

4. Boileau P. Complications and revision of reverse total shoulder arthroplasty. Orthop Traumatol Surg Res. 2016; 102(1 Suppl): S33-43.

5. Duramaz A, Bilgili MG, Bayram B, Ziroğlu N, Edipoğlu E, Öneş $\mathrm{HN}$, et al. The role of intraoperative cell salvage system on blood management in major orthopedic surgeries: a cost-benefit analysis. Eur J Orthop Surg Traumatol. 2018; 28(5): 991-7.

6. Copley LA, Richards BS, Safavi FZ, Newton PO. Hemodilution as a method to reduce transfusion requirements in adolescent spine fusion surgery. Spine. 1999; 24: 219-22.

7. Evangelista PJ, Aversano MW, Koli E, Hutzler L, Inneh I, Bosco J, et al. Effect of tranexamic acid on transfusion rates following total joint arthroplasty: a cost and comparative effectiveness analysis. Orthop Clin North Am. 2017; 48(2): 109-15.

8. George DA, Sarraf KM, Nwaboku H. Single perioperative dose of tranexamic acid in primary hip and knee arthroplasty. Eur J Orthop Surg Traumatol. 2015; 25: 129-33.

9. Day JS, Lau E, Ong KL, Williams GR, Ramsey ML, Kurtz SM. Prevalence and projections of total shoulder and elbow arthroplasty in the United States to 2015. J Shoulder Elbow Surg. 2010; 19: 1115-20.

10. Kim SH, Wise BL, Zhang Y, Szabo RM. Increasing incidence of shoulder arthroplasty in the United States. J Bone Joint Surg Am. 2011; 93: 2249-54.

11. Gillespie R, Shishani Y, Joseph S, Streit JJ, Gobezie R. Neer Award 2015: A randomized, prospective evaluation on the effectiveness of tranexamic acid in reducing blood loss after total shoulder arthroplasty. J Shoulder Elbow Surg. 2015; 24(11): 1679-84.

12. Friedman RJ, Gordon E, Butler RB, Mock L, Dumas B. Tranexamic acid decreases blood loss after total shoulder arthroplasty. J Shoulder Elbow Surg. 2016; 25: 614-8.

13. Vara AD, Koueiter DM, Pinkas DE, Gowda A, Wiater BP, Wiater JM. Intravenous tranexamic acid reduces total blood loss in reverse total shoulder arthroplasty: a prospective, double-blinded, randomized, controlled trial. J Shoulder Elb Surg. 2017; 26: 1383-9.

14. Pauzenberger L, Domej MA, Heuberer PR, Hexel M, Grieb A, Laky $\mathrm{B}$, et al. The effect of intravenous tranexamic acid on blood loss and early postoperative pain in total shoulder arthroplasty. Bone Joint J. 2017; 99-B(8): 1073-9.

15. Gruson KI, Accousti KJ, Parsons BO, Pillai G, Flatow EL. Transfusion after shoulder arthroplasty: an analysis of rates and risk factors. $J$ Shoulder Elbow Surg. 2009; 18: 225-30.

16. Kuo LT, Hsu WH, Chi CC, Yoo JC. Tranexamic acid in total shoulder arthroplasty and reverse shoulder arthroplasty: a systematic review and meta-analysis. BMC Musculoskelet Disord. 2018; 19(1): 60.

17. Anthony SG, Patterson DC, Cagle PJ Jr, Poeran J, Zubizarreta N, Mazumdar M, et al. Utilization and real-world effectiveness of tranexamic use in shoulder arthroplasty: a population-based study. $J$ Am Acad Orthop Surg. 2019; 27(19): 736-42.

18. Kim SH, Jung WI, Kim YJ, Hwang DH, Choi YE. Effect of tranexamic acid on hematologic values and blood loss in reverse total shoulder arthroplasty. Biomed Res Int. 2017; 2017: 9590803.

19. Abildgaard JT, McLemore R, Hattrup SJ. Tranexamic acid decreases blood loss in total shoulder arthroplasty and reverse total shoulder arthroplasty. J Shoulder Elbow Surg. 2016; 25: 1643-8.

Conflicto de intereses: Ninguno. 